\title{
Feasibility of low-dose infusion of alteplase for unsuccessful thrombolysis with urokinase in deep venous thrombosis
}

\author{
MAOFENG GONG, BOXIANG ZHAO, XU HE, JIANPING GU and GUOPING CHEN \\ Department of Interventional Radiology, Nanjing First Hospital, Nanjing Medical University, \\ Nanjing, Jiangsu 210006, P.R. China
}

Received February 18, 2018; Accepted July 31, 2019

DOI: $10.3892 /$ etm.2019.7938

\begin{abstract}
The aim of the present study was to investigate whether the rescue of thrombolysis with the recombinant tissue plasminogen activator (rt-PA) alteplase was an effective and safe therapeutic option in patients who did not respond to urokinase. Between February 2016 and February 2017, 26 patients with deep venous thrombosis (DVT) underwent rescue thrombolysis with alteplase. Unsuccessful thrombolysis with urokinase was defined as a lack of improvement in the degree of thrombotic removal with a lysis rate $<50 \%$ under one of the following three conditions: Two consecutive venography procedures, administration of $>3$ million units of total urokinase, or $>7$ days infusion duration. The thrombus score, lysis rate and post-thrombolysis safety of alteplase, following unsuccessful urokinase thrombolysis were all evaluated. At the end of the unsuccessful urokinase thrombolytic therapy, the mean duration of the perfusion was $6.09 \pm 1.60$ days, and the mean total dose was $(362.5 \pm 90.0) \times 10^{4}$ units. No significant difference was detected in the total thrombus score before $(7.85 \pm 2.40)$ and at the completion $(6.19 \pm 2.33)$ of urokinase thrombolysis $(\mathrm{P}>0.05)$. The mean duration of perfusion was 3.36 \pm 1.69 days, and the mean total infusion dose was $44.8 \pm 22.6 \mathrm{mg}$ for the rescue thrombolysis with alteplase. The mean thrombus score decreased to $1.19 \pm 2.10$ at the completion of rescue thrombolysis. The alteplase post-thrombolysis scores were significantly decreased compared with those of urokinase thrombolysis $(\mathrm{P}<0.05)$. There were $23(88.5 \%)$ patients who achieved a successful lysis rate (grade II/III) following rescue thrombolysis with alteplase, and symptoms of swelling and pain in the affected limbs were significantly improved. Successful thrombolysis rates in patients in the acute ( $<14$ days) and subacute (14-28 days) phases were high (93.3 and $81.8 \%$, respectively; $\mathrm{P}>0.05)$. No symptomatic pulmonary embolism
\end{abstract}

Correspondence to: Professor Guoping Chen, Department of Interventional Radiology, Nanjing First Hospital, Nanjing Medical University, 68 Changle Road, Nanjing, Jiangsu 210006, P.R. China E-mail: chenguoping_72@163.com

Key words: venous thrombosis, urokinase, alteplase, catheterdirected thrombolysis or major bleeding occurred during rescue thrombolysis, but minor bleeding complications occurred in 4 cases (15.4\%). In conclusion, rescue thrombolysis with alteplase led to an effective and safe outcome in patients with DVT who did not respond to initial thrombolysis with urokinase, and may be a valid and easy alternative treatment option.

\section{Introduction}

Deep vein thrombosis (DVT) of the inferior limbs is one of the most common diseases of the veins and it can develop into pulmonary embolism (PE) $(1,2)$. Compared with the use of anticoagulation alone, the use of thrombolysis for DVT not only breaks down blood clots but also has other advantages, such as early thrombus removal, reduced risk of post-thrombotic syndrome (PTS) and avoidance of DVT recurrence $(3,4)$. Thus, thrombolysis therapy is widely gaining favor and rapidly becoming popular in the endovascular treatment of DVT. To date, urokinase remains the most common fibrinolytic agent selected for use in Asian countries, on the basis of its economy and predictability for the treatment of DVT. However, there are certain limitations in its specificity and efficacy, and some patients do not respond to fibrinolytic therapy with urokinase (5).

At present, the further therapeutic treatment options for patients with DVT who do not respond to conventional fibrinolytic therapy with urokinase remain unclear. This is partly due to the difficulty of defining 'unsuccessful' thrombolysis with urokinase in this setting, whereas in patients with acute myocardial infarction or acute stroke, the criteria and consequences of unsuccessful thrombolysis are well established (6). In the setting of DVT, the recovery of clinical manifestations is a straightforward marker of thrombolytic efficacy, as well as a predictor of in-hospital course. Furthermore, a lower percentage of thrombotic removal grade and higher residual thrombotic burden after thrombolytic therapy have been shown to be adverse long-term outcomes of increased PTS risk (3). The optimization of early DVT revascularization could play a pivotal role in improving the immediate and long-term progression of patients with DVT who do not respond to thrombolysis with urokinase. However, there are few structured strategies for the rescue management of unsuccessful thrombolysis, and the most suitable management strategy for DVT patients who do not respond to thrombolysis has not been identified. 
The therapeutic options for patients who have undergone unsuccessful thrombolysis comprise different strategies including anticoagulation alone, repeat thrombolysis with recombinant tissue plasminogen activator (rt-PA) and other therapies. The majority of clinicians follow a conservative approach of anticoagulation alone and obtain an unsatisfactory outcome. Thrombolytic therapy is superior compared with anticoagulation alone (2-4). Alteplase is considered a common fibrinolytic agent of rt-PA and is used in Europe (5), thus, the present study aimed to investigate the efficacy and safety of rescue thrombolysis with alteplase in patients who do not respond to thrombolysis with urokinase based on a single-center institution.

\section{Materials and methods}

Patients. The protocol of the present retrospective study was approved by the Ethics Committee of Nanjing First Hospital (Nanjing, China; approval no. KY20140430-01-KS-01). Prior to therapy, informed consent was obtained from all patients likely to require endovascular treatment or their immediate family members. Prior to January 2016, urokinase was the exclusive fibrinolytic agent used at Nanjing First Hospital, and alteplase was used from January 2016. All patients had no contraindications to anticoagulation or thrombolysis therapy (7), and patients who met the following inclusion criteria were included in the study and received urokinase thrombolysis during the initial therapeutic encounter: Age, 18-75 years; proven recent DVT (7) (duration of symptoms $<28$ days); and a first-time objectively verified proximal DVT above mid-thigh level. Unsuccessful thrombolysis with urokinase was defined as a lack of improvement in the degree of thrombotic removal with a lysis rate $<50 \%$ under one of the following three conditions: Two consecutive venography procedures; administration of $>3$ million units of urokinase in total; or $>7$ days infusion duration. Patients with a higher risk of bleeding (7), such as those with a fibrinogen level (FIB) $<1.0 \mathrm{~g} / 1$, heparin-induced thrombocytopenia (HIT) or a life expectancy $<2$ years, were excluded.

Based on these criteria, 183 consecutive patients diagnosed with DVT were referred to the hospital between February 2016 and February 2017, and 145 (79.2\%) patients were treated with urokinase thrombolytic therapy in accordance with the institution's policy. A flowchart of the study is presented in Fig. 1. Thrombolysis with urokinase was considered unsuccessful in $28(19.3 \%)$ patients. Two ineligible patients were excluded from the present study as one patient had persistently low FIB levels $(<1.0 \mathrm{~g} / \mathrm{l})$ and the other experienced intracranial hemorrhage following the initial thrombolysis. The remaining 26 patients, who did not respond to initial urokinase thrombolytic therapy, received repeat catheter-directed thrombolysis (CDT) treatment with alteplase. The mean age of the patients was $50.7 \pm 17.2$ years (range, 22-75 years), and 14 (53.8\%) patients were female. The onset of symptoms occurred $\leq 14$ days earlier in $\sim 57.7 \%$ of the patients (acute phase), and between 14 and 28 days in the remainder (subacute phase). Locations of the thrombus were the left lower limb (17 patients) or the right lower limb (9 patients); none were bilaterally located. Ten (38.4\%) patients had no risk factors for DVT, and 15 patients $(50 \%)$ had transient risk factors, such as surgery, trauma, short-term immobility and pregnancy. Three (11.6\%) patients had permanent risk factors, including cancer and obesity, for DVT (3).

Procedure. The initial diagnosis of DVT was verified by medical history, physical examination and compression ultrasound. If this was inconclusive, supplementary venography was also conducted. In accordance with local routines based on international guidelines $(2,7)$, anticoagulant treatment was initiated on the same day with the use of subcutaneous low molecular weight heparin (LWMH; Hebei Changshan Biochemical Pharmaceutical Co., Ltd.) at a bolus dose of $100 \mathrm{U} / \mathrm{kg}$ per $12 \mathrm{~h}(2)$.

A temporary filter was inserted into the inferior vena cava (IVC) of patients with an extensive proximal venous thrombus that was evaluated as potentially life-threatening; the filter was inserted via the femoral vein of the non-affected leg prior to the next treatment step (8). Based on the location and distribution of the thrombus, the visibility of the popliteal vein by venography, and the tolerance of the patient to the prone position, a retrograde approach using contralateral femoral vein access or an anterograde approach via the lateral popliteal vein in the affected leg was selected. After local anesthesia, a 5-6-French $(F)$ sheath was inserted into the femoral vein or popliteal vein. Subsequently, the H1 catheter (Cordis Corporation) and a 0.035-inch smooth guide-wire (Terumo Corporation) was passed through the thrombus segment and advanced up to the IVC. Next, the H1 catheter was exchanged for a multiple side hole catheter (Cook Medical LLC). Repeat venography from the popliteal vein to the IVC was then conducted to assess the location and severity of the thrombus and ensure that the catheter was positioned in the true lumen. For patients estimated to have an extensive proximal venous thrombus, adjunctive endovascular techniques, including aspiration thrombectomy (the use of a syringe to aspirate the thrombus from the vein via a catheter, device or sheath) or balloon maceration (the use of an angioplasty balloon to macerate or fragment the thrombus), were used to enlarge and maintain the venous lumen patency (7).

Subsequently, a 4-5F perfusion catheter (Uni*Fuse infusion catheter; AngioDynamics,) of appropriate-length and with multiple side holes spanning 10-50 $\mathrm{cm}$, was inserted according to the length of the residual thrombotic segments. Catheter-directed infusion of urokinase (Livzon Pharmaceutical Group, Inc.) was then established. According to the patient's body constitution, the amount of thrombus and blood coagulation status, the specific dosage regimen given was as follows (9): 25-75 million units of urokinase in $500 \mathrm{ml} 0.9 \%$ normal saline, at a steady infusion rate of 2-3 million $U / h$. The patients were confined to bed with the affected limb raised at an upward angle of $30^{\circ}$ during the administration of the thrombolytic infusion, and routine blood tests were monitored daily. Repeat venography examination was systematically performed to monitor the progression of thrombolysis every $48 \mathrm{~h}$ after the thrombolytic therapy. The position of the perfusion catheter was adjusted if necessary to ensure that the infusion section of the catheter was deeply buried in the thrombus. The patients were also instructed to make ankle joint-toe movements.

When unsuccessful thrombolysis with urokinase was identified, the thrombolytic therapy was adjusted. Urokinase 


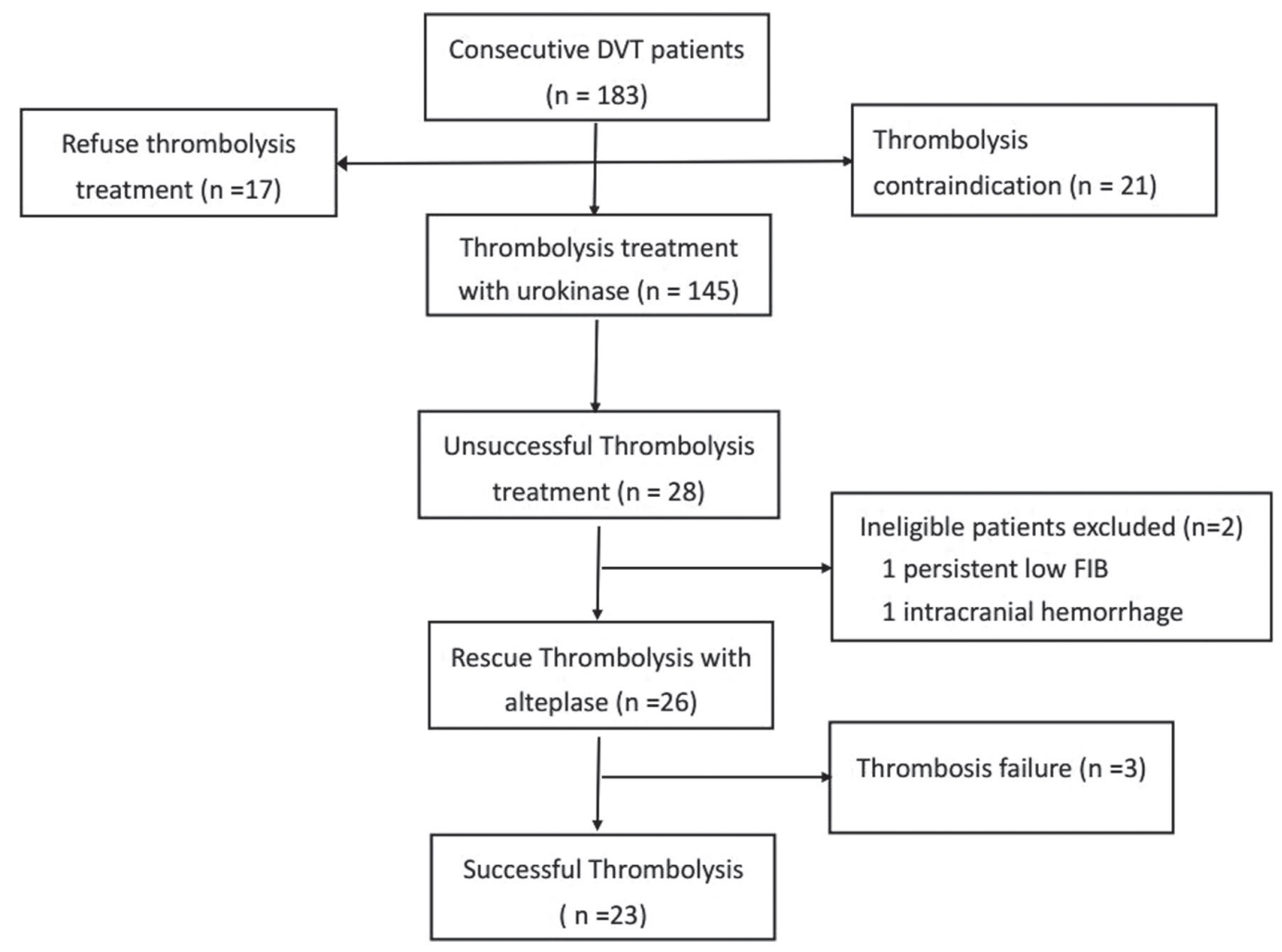

Figure 1. Flowchart of the study design, demonstrating the number of patients who underwent unsuccessful thrombolysis with urokinase and rescue thrombolysis with alteplase treatment. DVT, deep venous thrombosis; FIB, fibrinogen level.

infusion through the catheter was discontinued immediately, and the patients without exclusion criteria were subsequently treated with a continuous infusion of the rt-PA alteplase (Actilyse ${ }^{\circledR}$; Boehringer Ingelheim International $\mathrm{GmbH}$ ). The alteplase thrombolytic therapy was administered as $20 \mathrm{mg}$ alteplase in $500 \mathrm{ml} 0.9 \%$ normal saline via the catheter at an infusion rate of $0.01 \mathrm{mg} / \mathrm{kg} / \mathrm{h}$; the maximum rate was $\leq 1.0 \mathrm{mg} / \mathrm{h}$. Both urokinase and alteplase were only administered when the FIB level was $>1 \mathrm{~g} / \mathrm{l}$.

Other comprehensive interventional treatment methods were the same as reported previously by Chen et al (9) and Shi et al (10). Following CDT, an 8-14-mm diameter balloon catheter (C.R. Bard, Inc.) angioplasty was performed when the residual stenosis rate of the iliac vein was assessed to be $>50 \%$. Subsequently, a self-expandable stent (12-14 mm; Luminexx, $\mathrm{CR}$, Inc.) was deployed in cases with residual stenosis lesion rates $>50 \%$ after balloon angioplasty (10). After stent implantation, if the consecutive venography showed a coarse lumen and relatively slow blood flow within the lumen of the stent, a perfusion catheter was maintained to continue thrombolysis for $\geq 24 \mathrm{~h}$. If thrombolysis treatment was terminated and patients' symptoms, such as swelling of the limb, could not be remitted, physical massage treatment (pressure, $60-80 \mathrm{mmHg}$ ) was applied using an air wave pressure circulation therapy instrument (Power-Q3000; Wonjin Mulsan Co., Ltd.) for 30 min each time, once or twice each day. During each thrombolysis therapy, LMWH therapy overlapped with oral warfarin therapy was used for 3-5 days. The warfarin dose was adjusted thereafter to maintain the international normalized ratio at 2.0-3.0 for $\geq 6$ months (11). After discharge from the hospital, patients were advised to wear graded-compression stockings with an ankle pressure of $30-40 \mathrm{mmHg}(1 \mathrm{mmHg}=0.133 \mathrm{kPa})$ for $\geq 2$ years (12).

Outcomes and safety. A total thrombus score was calculated independently through venography by three experienced interventional physicians before and at the completion of thrombolysis by adding the scores of seven vein segments (IVC, common iliac vein, external iliac vein, common femoral vein, proximal and distal segments of femoral vein, and popliteal vein) (13). Thrombus scores were determined as follows: 0, vein was patent and completely free of thrombus; 1 , partially occluded vein; and 2, completely occluded vein (vein lumen completely occluded with massive thrombus). The score was calculated for each segment, resulting in a possible total thrombus score of $0-14$. The primary efficacy of thrombolysis was classified based on post-lysis thrombus scores and the thrombus lysis grade at the completion of the procedure. The lysis grade was calculated by dividing the difference between the total pre- and post-lysis thrombus scores by the pre-lysis score, resulting in grade III (100\% lysis with no residual clots), grade II (50-99\% lysis), and grade I ( $<50 \%$ lysis). Lysis grades II and III ( $\geq 50 \%$ lysis) were considered successful outcomes $(13,14)$.

The complications that occurred during CDT included major complications, such as a reduction of hemoglobin level to $2 \mathrm{~g} / \mathrm{dl}$ (requiring transfusion of $\geq 2$ units of packed red blood cells), intracranial hemorrhage, massive hemorrhage of other critical organs that could lead to death, or PE. Minor complications included bleeding and hematoma at the puncture site, gross or microscopic hematuria, mucosal bleeding and other small bleeding events and infections, which could be controlled by simple compression or stopping the thrombolysis $(14,15)$. 
Table I. Indications for alteplase substitution of urokinase in 26 patients with DVT.

Conditions after thrombolysis with urokinase

No. of patients $(\%)$

Insoluble or aggravated severe symptoms after $48 \mathrm{~h}$

$16(61.5)$

Thrombus extension

To the popliteal-femoral vein

To the iliac-femoral vein

To the IVC

Lack of improvement in thrombotic removal grade, lysis rate $<50 \%$

$16(61.5)$

$>3$ million units or infusion time $>7$ days, lysis rate $<50 \%$

$17(65.4)$

Subacute DVT underwent 48 -h CDT, lysis rate $<50 \%$

$11(42.3)$

DVT, deep venous thrombosis; CDT, catheter-directed thrombolysis; IVC, inferior vena cava.

Statistical analysis. The SPSS statistical package (version 23.0; SPSS Corp.) was used for all statistical analyses in the present study. Continuous variables are expressed as the mean \pm standard deviation. Qualitative variables are presented as a percentage. When assessing the difference between pre-procedural and post-procedural variables, a paired t-test was used. Significance for qualitative variables was tested with a Fisher's exact test. $\mathrm{P}<0.05$ was used to indicate a statistically significant difference.

\section{Results}

Clinical characteristics of patients and urokinase thrombolysis. All the patients initially showed typical clinical manifestations of DVT, such as pain, swelling and/or activity limitation in the affected limb. The clinical characteristics of the patients in whom the urokinase thrombolysis was unsuccessful are presented in Table I. After initial urokinase thrombolytic therapy for $48 \mathrm{~h}, 16(61.5 \%)$ patients experienced insoluble initial symptoms, and 5 (19.2\%) patients manifested aggravated swelling in the affected limb. Subsequent repeat venography showed extension of the clots through the popliteal femoral vein, iliac-femoral vein and the IVC. There were $16(61.5 \%)$ patients with no further improvement in thrombotic removal degree, and the lysis rate of $17(65.4 \%)$ patients remained $<50 \%$ despite a total dose of $>3$ million units of urokinase or an infusion time $>7$ days. Additionally, all 11 patients in the subacute phase (14-28 days) underwent urokinase thrombolytic therapy for $48 \mathrm{~h}$, but the lysis rate remained $<50 \%$. At the end of unsuccessful urokinase thrombolytic therapy, the mean duration of the perfusion was $6.09 \pm 1.60$ days, and the total dose was $(362.5 \pm 90.0) \times 10^{4}$ units. The total thrombus scores calculated before and at the completion of thrombolysis were $7.85 \pm 2.40$ and $6.19 \pm 2.33$, respectively. The post-lysis scores were not significantly decreased compared with those before urokinase thrombolysis $(\mathrm{P}>0.05)$.

Rescue thrombolysis with alteplase and outcomes. All 26 patients subsequently received rescue thrombolysis with alteplase. The mean duration of the perfusion was $3.36 \pm 1.69$ days, and the mean total infusion dose was
$44.8 \pm 22.6 \mathrm{mg}$. The mean thrombus scores decreased to $1.19 \pm 2.10$ at completion. The post-lysis scores were significantly reduced compared with those at the end of urokinase thrombolysis $(\mathrm{P}<0.05)$. Successful lysis (grade II/III) of rescue thrombolysis with alteplase was obtained in $23(88.5 \%)$ patients, and the symptoms of swelling and pain in the affected limbs were significantly improved. The venography results of a typical case are shown in Fig. 2. Based on the symptom duration, successful lysis was achieved in the majority of patients in the acute phase $(93.3 \%)$ and subacute phase $(81.8 \%)$. No statistically significant differences were detected between the groups $(\mathrm{P}>0.05)$, and the lysis rates were high in both groups. The distributions of lysis grade are presented in Table II.

Adjunctive therapies. All 20 patients with DVT evaluated as potentially life-threatening received a recoverable IVC filter ( 8 cases used a Celect ${ }^{\mathrm{TM}}$ filter, Cook Group, Inc.; 8 cases used an Optease filter, Cordis; Cardinal health, Inc.; and 4 cases used an Aegisy ${ }^{\mathrm{TM}}$ filter, LifeTech Scientific Corporation) prior to interventional treatment, and one underwent mechanical thrombectomy with an AngioJet device during urokinase thrombolysis. While rescue thrombolysis with alteplase was discontinued, filters were retrieved from 19 (95.0\%) patients, and one was laid permanently because of superior paraplegia. Adjunctive angioplasty was performed in 15 patients (57.7\%); only $4(26.7 \%)$ patients underwent dilation with a balloon in the iliac vein, among whom 2 patients also had balloon dilatation in the femoral vein. Stents (average diameter $13.5 \mathrm{~mm}$, range $12-14 \mathrm{~mm}$ ) were inserted into the iliac veins of $4(15.4 \%)$ patients.

Safety of rescue thrombolysis with alteplase. No symptomatic $\mathrm{PE}$ or major complications occurred during ongoing rescue thrombolysis with alteplase. A total of 8 complications were reported, of which $4(15.7 \%)$ were classified as minor complications. Three of the four bleeding complications were restricted to bleeding at the puncture site, all of which occurred in inguinal punctures, and one case of gingival bleeding was reported. The FIB level of 4 patients (15.7\%) was reduced to $1.0 \mathrm{~g} / \mathrm{l}$, and in $3(75 \%)$ cases this reduction occurred within the third day of rescue thrombolysis with alteplase. Thus, the infusion rate was suspended until the FIB level recovered 
A

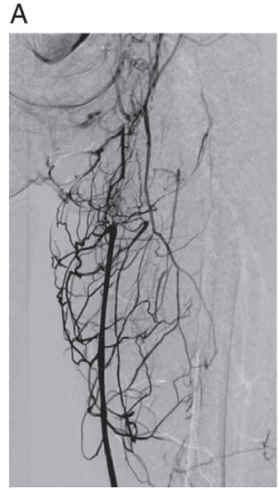

E

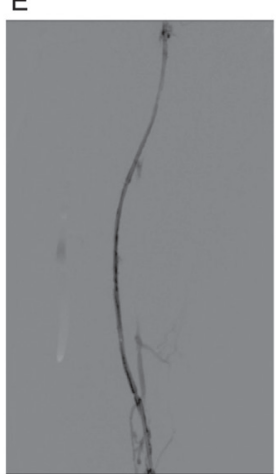

B

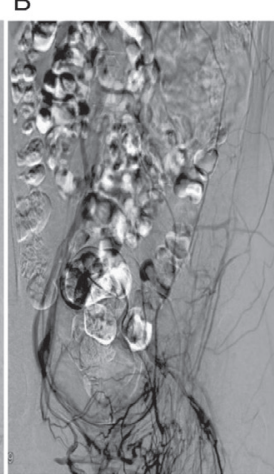

F

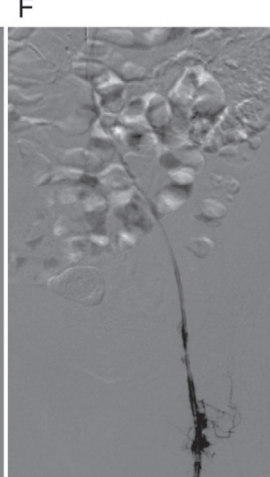

C
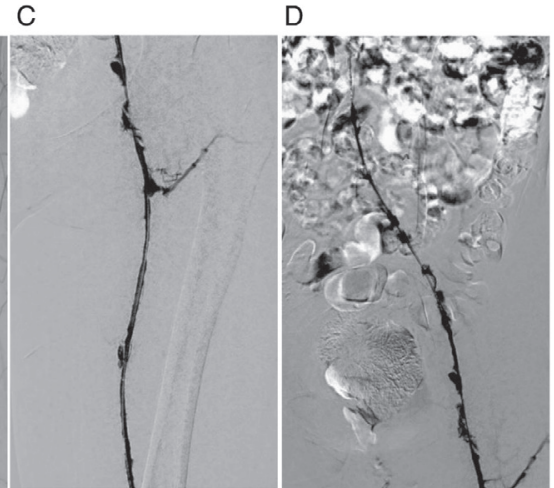

G

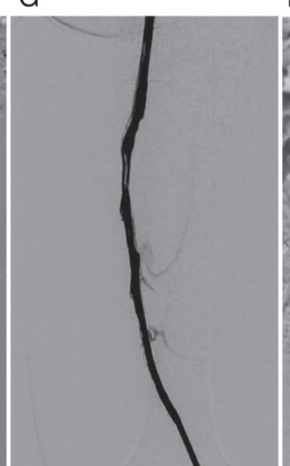

$\mathrm{H}$

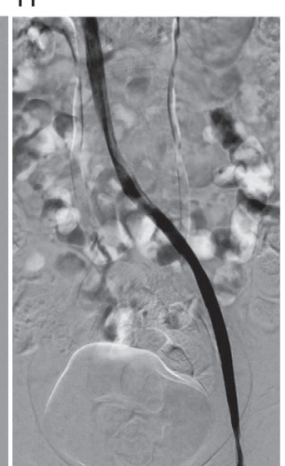

Figure 2. Venography of a 23-year-old woman with lower left-limb deep venous thrombosis. (A and B) Venography from the dorsalis pedis vein indicated that all of the trunk lumens from the left popliteal vein to the common iliac vein were occlusive, and massive collateral vessels are shown. (C and D) Repeat venography $48 \mathrm{~h}$ after thrombolysis with urokinase via the infusion catheter revealed the superficial femoral vein and irregular iliac-femoral vein with resolution of thrombosis. (E and F) Another $24 \mathrm{~h}$ after thrombolysis with urokinase, the repeat venography confirmed a lack of further improvement in thrombotic removal degree, and apparent worsening compared with its former condition. ( $\mathrm{G}$ and $\mathrm{H}$ ) Alteplase was substituted with urokinase for continuous $36 \mathrm{~h}$ infusion, and venography revealed complete patent blood flow.

to $>1.0 \mathrm{~g} / 1$, and 1 patient was treated with cryoprecipitated antihemophilic factor transfusion.

\section{Discussion}

The risk factors of DVT vary and are largely dependent upon various conditions of the individual, such as age (older age, $>65$ years), obesity, hypertension, metabolic syndrome, cigarette smoking and protein $\mathrm{C}$ or $\mathrm{S}$ deficiency, or are secondary to malignant tumor, major surgery, immobilization, pregnancy, trauma, oral contraceptives and acute medical illness, such as pneumonia or congestive heart failure (9). The traditional anticoagulation therapy for DVT is considered a 'non-aggressive' routine approach to avoid the propagation of clots and prevent symptomatic PE, and can be reserved for patients with contraindications of thrombolysis (16). However, anticoagulation alone depends primarily on the effectiveness of the natural fibrinolytic system (1). Incomplete thrombus resolution causes inflammatory responses, provoking severe compromised valvular competency, resulting in impaired venous reflux and/or venous insufficiency and ultimately leading to ambulatory venous hypertension (16). Hence, $70.8 \%$ of patients from the Catheter-directed Venous Thrombolysis in Acute Iliofemoral Vein Thrombosis (CaVenT) study developed some degree of PTS within 5 years (3). Studies have demonstrated that additional CDT can accelerate clot lysis, partially or completely restore the blood flow of tissues and/or organs, relieve venous hypertension, and in time preserve existing valve function $(3,16)$. PTS has been demonstrated to achieve an absolute risk reduction of $28 \%$ in 5 years (3). Therefore, CDT is administered as an 'aggressive' and effective approach for DVT. Clinically, for fear of increased hemorrhagic complication risk, the majority of institutions in China terminate urokinase usage if the total dose of urokinase exceeds $300-400$ units or the FIB level is $<1.0 \mathrm{~g} / 1$ in patients with a residual thrombus burden. The CaVenT findings indicate that the percentage of residual thrombus was positively associated with the incidence of PTS $(2,3)$, thus, the prognosis of patients who do not respond to thrombolysis with urokinase appeared to be poor. Thus, it is reasonable for patients who do not respond to thrombolysis with urokinase to subsequently receive rescue thrombolysis. No peer-reviewed data exploring the management of unsuccessful thrombolysis with established urokinase protocols have been published. Based on this, urokinase was replaced with alteplase in patients who did not respond to urokinase in the present study, with the aim of increasing the successful lysis rate and promoting venous lumen patency.

The successful lysis rates of urokinase and alteplase reported in multiple central large-scale comparative clinical trials, such as the National Venous Registry (13) and CaVenT (4), were 83.0 and $88.9 \%$, respectively. However, because these lysis rates were collected from two non-uniform studies, some differences were present in the inclusion criteria. Thus, it is difficult to accurately determine the therapeutic efficacy of the two fibrinolytic agents on the basis of these two superficial rates alone. A previous study reported the successful lysis rate 
Table II. Comparison of rescue thrombolysis efficacy according to phase of symptom onset.

Lysis grade, $\mathrm{n}(\%)$

\begin{tabular}{lccccc}
\cline { 3 - 5 } Group & No. of cases & Grade I & Grade II & Grade III & Rate of grade II or III lysis (\%) \\
\hline Acute phase & 15 & $1(6.7)$ & $2(13.3)$ & $12(80)$ & 93.3 \\
Subacute phase & 11 & $2(18.2)$ & $3(27.3)$ & $6(54.5)$ & 81.8
\end{tabular}

Acute phase, symptom onset $<14$ days; subacute phase, symptom onset 14-28 days. Significance was tested with a Fisher's exact test. P $>0.05$ for acute phase vs. subacute phase.

of a low-dose urokinase infusion method as $82.2 \%$ (9), which was consistent with the results of the aforementioned two studies. The lack of success of thrombolysis with urokinase in this group may be attributable to several factors. Firstly, reduced pharmacological efficacy of urokinase may be a contributing factor to unsuccessful thrombolysis; the specification of urokinase suggests it should be used within $8 \mathrm{~h}$, at a temperature of $25^{\circ} \mathrm{C}$, and if the temperature is $2-5^{\circ} \mathrm{C}$ its preservation can be prolonged to $48 \mathrm{~h}$. In the present study, urokinase was unused for an extended time ( $24 \mathrm{~h})$; hence, its pharmacological efficacy may have been reduced. Secondly, existing differences in individual sensitivity may also explain unsuccessful thrombolysis with urokinase. Thirdly, the relatively poor local clot infiltration effect of urokinase may also be a factor, especially for partially underlying non-acute phase clots or abnormalities refractory to thrombolysis with urokinase. Even so, this result is not applicable to all patients, as a previous report indicated that urokinase can sometimes be effective for non-acute phase patients (17). Furthermore, a lack of positive blood flow into the thrombus, caused by time-dependent structural changes of the thrombus that may impede the agent from flowing through the clotted segment where the partial accumulation of the thrombus is incomplete, can also lead to unsuccessful thrombolysis. Patients benefitted from timely relief of the outflow obstruction first and then continued with thrombolysis; treatment of residual stenosis after thrombolysis appears to be more reasonable, although it has not been recommended in the literature. Several factors are associated with the lysis rate of thrombus, such as the duration of thrombolysis, doses of lysis-inducing agents and the mode of delivery. However, a previous study reported that the proportion of patients in which a lysis rate $\geq 50 \%$ was achieved reached a maximum of $82.8 \%$, when mean doses of 3-4 million units and an infusion time of $<7$ days were used (9). In the present study protocol, the duration and doses of treatment used in some patients who were unresponsive to initial thrombolysis might not have reached the threshold of thrombus lysis. Therefore, extending thrombolysis beyond this dose and increasing the time frame may improve the lysis grade; however, it may also result in a higher risk of bleeding.

During thrombolysis with urokinase, the lysis thrombus scores pre- $(7.85 \pm 2.40)$ and post-thrombolysis $(6.19 \pm 2.33)$ treatment for all 26 patients exhibited no significant difference $(\mathrm{P}>0.05)$. As the CaVenT study indicated, disregarding the pre-lysis score, the post-lysis score was inversely correlated with thrombosis degree and patency during follow-up, which was clearly correlated with a reduction in the frequency of
PTS (14). Rescue thrombolysis with alteplase had a mean duration of $3.36 \pm 1.69$ days, decreased the lysis thrombus score to $1.19 \pm 2.10(\mathrm{P}<0.05)$ and achieved a high mean clot resolution of $88.5 \%$ in the in-hospital patients. At the same time, the clinical symptoms and signs of onset were both ameliorated. It is predicted that these patients may have a lower incidence of PTS and better prognosis, but the long-term efficacy requires further follow-up and investigation. However, all the present findings indicated that rescue thrombolysis with alteplase might be considered an effective therapeutic option in patients with unsuccessful thrombolysis. The highly successful lysis result observed in patients with rescue thrombolysis may be attributed to the two circular structures (K region: Ligand binding site) in the molecular structure of alteplase, which allow it to selectively bind to a specific lysine site at the thrombus surface, thereby specifically activating fibrinogen at a local area of the thrombus and providing selective infiltrative thrombolysis (5). Further sub-analysis, according to the time of symptom onset in the subgroups, revealed that thrombolysis with alteplase can obtain satisfactory clinical efficacy in the subacute phase that is comparable with that in the acute phase $(\mathrm{P}>0.05)$; this may expand the conclusion reported by Sugimoto et al (18).

The incidences of major and minor bleeding complications in the thrombolytic procedure have been reported to be 16.0 and $11.0 \%$ for urokinase (13) and 21.7 and 3.3\% for alteplase (2). However, an assessment of the safety of rescue thrombolysis with alteplase following urokinase has not been reported in other studies. During the remedial thrombolysis with alteplase, no symptomatic PE or major bleeding complications occurred, and the rate of minor bleeding complications was only $15.7 \%$. Therefore, compared to a previous report using urokinase alone, additional rescue thrombolysis with alteplase for unsuccessful thrombolysis with urokinase did not increase the bleeding risk (9). However, as the present study was limited by the small number of cases, this conclusion requires validation in controlled studies. In addition, $15.7 \%$ of patients during rescue thrombolysis with alteplase experienced reduced FIB $(<1.0 \mathrm{~g} / \mathrm{l})$ the present study; however, additional bleeding complications did not increase. Alteplase has a fast fibrinogen-lowering effect, but due to its short half-life (4-8 $\mathrm{min}$ ), it is safe to use (5).

The overall infusion methods of CDT for DVT are diverse, consisting of continuous infusion (14), pulse-spray infusion (19) and mixed-type infusion (20), comprising a combination of continuous and pulse-spray infusions To date, there is no convincing clinical evidence in current guidelines that is superior to others. In addition, data on the optimal infusion method for rescue thrombolysis are lacking, and 
the optimal pattern remains under debate. Alteplase has a half-life of 4-8 min in the plasma and decreases the residual circulating fibrinogen levels to $<10 \%$ of the initial value for $\sim 20 \mathrm{~min}$, resulting in reduced efficacy (5). To maintain a sustained drug concentration, the preferred method in the present study was to continuously infuse alteplase directly into clots by an infusion pump through catheters embedded into clots, similar to the CaVenT study (3). Although current guidelines have no consensus on the optimal rates and doses of infusion in thrombolysis with alteplase, the increased experience of clinical alteplase application has led to a gradual reduction in the doses of alteplase being administered. Due to the need for protocols for the use of alteplase in thrombolysis, the Society of Cardiovascular and Interventional Radiology first published guidelines recommending the use of alteplase at a 'low dose' of $\leq 2 \mathrm{mg} / \mathrm{h}$ (18), and in 2009, the Society of Interventional Radiology (SIR) and European Cardiovascular Interventional Radiology (CIR) recommended that the dose should be decreased to $0.5 \mathrm{mg} / \mathrm{h}$ (16). To date, the current guidelines recommended a weight-based regimen at the rate of $0.01 \mathrm{mg} / \mathrm{kg} / \mathrm{h}$, with a maximum of $1.0 \mathrm{mg} / \mathrm{h}(10,11)$. This recommendation was applied in the present study and resulted in satisfactory efficacy and safety.

At present, there is a lack of formal large-scale comparative clinical trials to confirm the time window during which to carry out rescue thrombolysis, which is important for successful thrombolysis (14). Based on the observations of the present study, the following is suggested. Firstly, if the thrombus is in a non-acute phase and/or patients have undergone unsuccessful thrombolysis for $>48 \mathrm{~h}$, the sensitivity of the thrombus to urokinase needs to be considered. In order to shorten the time of thrombolysis and to salvage valve function, alteplase was substituted for urokinase in the present study when thrombolysis was continued. The results support the recommendation of alteplase as the first-line fibrinolytic agent for patients with subacute DVT. Secondly, if the pharmacoeconomic factors of alteplase are taken into consideration, urokinase may be used first for patients who have difficulty affording alteplase, but the fibrinolytic agent must be changed immediately if there is no change in the thrombolysis removal grade during two consecutive venography procedures, especially if the thrombus extends to the IVC and the longest retrieval time of the IVC filter is near. Thirdly, if a patient's symptoms exhibit no obvious improvement within $48 \mathrm{~h}$ of the initial thrombolysis with urokinase, or are further aggravated, alteplase may be used as a remedy.

The conclusions that may be drawn from the present study are limited because it was a small, retrospective, non-randomized analysis from a single center, and the causes of unsuccessful thrombolysis were partially based on a hypothesis from experience. Nevertheless, to the best of our knowledge, this is the first study to use alteplase following unsuccessful thrombolysis with urokinase. Additionally, a gold standard diagnostic method for unsuccessful thrombolysis is still lacking. The present study was also constrained by the use of parameters (thrombotic removal degree, infusion time and infusion dose, which are considered straightforward markers of thrombolytic efficacy) that were selected for defining patients with unsuccessful thrombolysis. Furthermore, the study was limited by the lack of follow-up outcomes for estimating the long-term efficacy of rescue thrombolytic therapy. Therefore, it should be regarded as a preliminary study for assessment of the instant efficacy of treatment, and further follow-up studies are required.

In conclusion, the aim of the present study was to accelerate the vein recanalization process in an attempt to save the valve function and thereby minimize the risks of PTS (21). Rescue thrombolysis with alteplase was feasible in most patients and led to an effective and secure result in the management of patients with DVT who did not respond to initial thrombolysis with urokinase. This may, therefore, be considered a valid and easy alternative treatment, which may accelerate the process of recanalization of the vein and lead to a better in-hospital course. However, further confirmation of the conclusions using long-term analyses is necessary.

\section{Acknowledgements}

Not applicable.

\section{Funding}

Funding was received from the Clinical Medicine Science and Technology Projects of Jiangsu Province, China (grant. no. BL2014013) and the Nanjing Medical Science Fund, China (grant. no. YKK14087).

\section{Availability of data and materials}

The datasets used and/or analyzed during the present study are available from the corresponding author on reasonable request.

\section{Authors' contributions}

MG, BZ, XH, JG and GC contributed to the conception and design of the study. MG and BZ were involved in data collection. MG, BZ and GC contributed to data analysis, interpretation, statistical analysis and writing the manuscript. $\mathrm{MG}, \mathrm{XH}$ and $\mathrm{GC}$ revised the manuscript. All authors read and approved the final manuscript.

\section{Ethics approval and consent to participate}

The present study was approved by the Ethics Committee of Nanjing First Hospital (Nanjing, China).

\section{Patient consent for publication}

Not applicable.

\section{Competing interests}

The authors declare that they have no competing interests.

\section{References}

1. Kearon C: Natural history of venous thromboembolism. Circulation 107 (Suppl 1): S122-S130, 2003.

2. Kearon C, Akl EA, Ornelas J, Blaivas A, Jimenez D, Bounameaux H, Huisman M, King CS, Morris TA, Sood N, et al: Antithrombotic therapy for VTE disease: CHEST guideline and expert panel report. Chest 149: 315-352, 2016. 
3. Haig Y, Enden T, Grøtta O, Kløw NE, Slagsvold CE, Ghanima W, Sandvik L, Hafsahl G, Holme PA, Holmen LO, et al: Post-thrombotic syndrome after catheter-directed thrombolysis for deep vein thrombosis (CaVenT): 5-year follow-up results of an open-label, randomised controlled trial. Lancet Haematol 3: 64-71, 2016.

4. Watson L, Broderick C and Armon MP: Thrombolysis for acute deep vein thrombosis. Cochrane Database Syst Rev: Nov 10 2016 (Epub ahead of print). doi: 10.1002/14651858.CD002783. pub3.

5. Gurman P, Miranda OR, Nathan A, Washington C, Rosen Y and Elman NM: Recombinant tissue plasminogen activators (rtPA): A review. Clin Pharmacol Ther 97: 274-285, 2015.

6. Meneveau N, Seronde MF, Blonde MC, Legalery P, Didier-Petit K, Briand F, Caulfield F, Schiele F, Bernard Y and Bassand JP. Management of unsuccessful thrombolysis in acute massive pulmonary embolism. Chest 129: 1043-1050, 2006.

7. Vedantham S, Sista AK, Klein SJ, Nayak L, Razavi MK, Kalva SP, Saad WE, Dariushnia SR, Caplin DM, Chao CP, et al: Quality improvement guidelines for the treatment of lower-extremity deep vein thrombosis with use of endovascular thrombus removal. J Vasc Interv Radiol 25: 1317-1325, 2014.

8. Decousus H, Leizorovicz A, Parent F, Page Y, Tardy B, Girard P, Laporte S, Faivre R, Charbonnier B, Barral FG, et al: A clinical trial of vena caval filters in the prevention of pulmonary embolism in patients with proximal deep-vein thrombosis. Prévention du Risque d'Embolie Pulmonaire par Interruption Cave Study Group. N Engl J Med 338: 409-415, 1998.

9. Chen G, Shi W, He X, Lou W, Chen L and Gu J: Feasibility of continuous, catheter-directed thrombolysis using low-dose urokinase in combination with low molecular-weight heparin for acute iliofemoral venous thrombosis in patients at risk of bleeding. Exp Ther Med 13: 751-758, 2017.

10. Shi WY, Gu JP, Liu CJ, He X and Lou WS: Endovascular treatment for iliac vein compression syndrome with or without lower extremity deep vein thrombosis: A retrospective study on mid-term in-stent patency from a single center. Eur J Radiol 85: 7-14, 2016.

11. Farraj RS: Anticoagulation period in idiopathic venous thromboembolism. How long is enough? Saudi Med J 25: 848-851, 2004

12. Kahn SR, Shapiro S, Wells PS, Rodger MA, Kovacs MJ, Anderson DR, Tagalakis V, Houweling AH, Ducruet T, Holcroft C, et al: Compression stockings to prevent post-thrombotic syndrome: A randomised placebo-controlled trial. Lancet 383 : 880-888, 2014
13. Mewissen MW, Seabrook GR, Meissner MH, Cynamon J, Labropoulos N and Haughton SH: Catheter-directed thrombolysis for lower extremity deep venous thrombosis: Report of a national multicenter registry. Radiology 211: 39-49, 1999.

14. Haig Y, Enden T, Slagsvold CE, Sandvik L, Sandset PM and Kløw NE: Determinants of early and long-term efficacy of catheter-directed thrombolysis in proximal deep vein thrombosis. J Vasc Interv Radiol 24: 17-24, 2013

15. Buller HR, Davidson BL, Decousus H, Gallus A, Gent M, Piovella F, Prins MH, Raskob G, van den Berg-Segers AE, Cariou $\mathrm{R}$, et al: Subcutaneous fondaparinux versus intravenous unfractionated heparin in the initial treatment of pulmonary embolism. N Engl J Med 349: 1695-1702, 2003.

16. Vedantham S, Piazza G, Sista AK and Goldenberg NA: Guidance for the use of thrombolytic therapy for thetreatment of venous thromboembolism. J Thromb Thrombolysis 41: 68-80, 2016.

17. Gao B, Zhang J, Wu X, Han Z, Zhou H, Dong D and Jin X: Catheter-directed thrombolysis with a continuous infusion of low-dose urokinase for non-acute deep venous thrombosis of the lower extremity. Korean J Radiol 12: 97-106, 2011.

18. Sugimoto K, Hofmann LV, Razavi MK, Kee ST, Sze DY, Dake MD and Semba CP: The safety, efficacy, and pharmacoeconomics of low-dose alteplase compared with urokinase for catheter-directed thrombolysis of arterial and venous occlusions. J Vasc Surg 37: 512-517, 2003.

19. Chang R, Chen CC, Kam A, Mao E, Shawker TH and Horne MK III: Deep vein thrombosis of lower extremity: Direct intraclot injection of alteplase once daily with systemic anticoagulation-results of pilot study. Radiology 246: 619-629, 2008.

20. Manninen H, Juutilainen A, Kaukanen E and Lehto S: Catheter-directed thrombolysis of proximal lower extremity deep vein thrombosis: A prospective trial with venographic and clinical follow-up. Eur J Radiol 81: 1197-1202, 2012.

21. Fiengo L, Bucci F, Khalil E and Salvati B: Original approach for thrombolytic therapy in patients with Ilio-femoral deep vein thrombosis: 2 years follow-up. Thromb J 13: 40, 2015. 